International Journal of Pure and Applied Mathematics

Volume 106 No. 1 2016, 101-113

ISSN: 1311-8080 (printed version); ISSN: 1314-3395 (on-line version)

url: http://www.ijpam.eu

doi: 10.12732/ijpam.v106i1.8

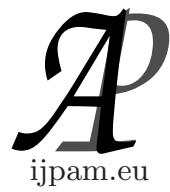

\title{
KRASNER TERNARY HYPER FIELDS AND MORE \\ CHARACTERIZATION OF PRIME AND MAXIMAL HYPER IDEALS IN KRASNER TERNARY HYPER RINGS
}

\author{
Jovannie R. Castillo \\ Mathematics Department \\ College of Arts and Sciences \\ La Salle University \\ Ozamiz City, 5200, PHILIPPINES
}

\begin{abstract}
In 2010, Davvaz and Mirvakili introduced a new class of hyper structure called an $(m, n)$-Krasner hyperring, constructed its quotient class where the hyper ideal considered in the said construction is normal, and proved the isomorphism theorems. Recently, Castillo and Vilela investigated the (2,3)-Krasner hyperring called a Krasner ternary hyperring, and constructed its quotient class via another relation where the hyper ideal considered in the construction is not necessarily normal. In this article, a Krasner ternary hyper field is defined and characterized. Moreover, more structure results on prime and maximal hyper ideals not found in [1] are established.
\end{abstract}

AMS Subject Classification: 03E20, 05C25

Key Words: Krasner ternary hyperring, Krasner $(m, n)$-hyperring

\section{Introduction}

Hyperstructure theory was introduced in 1934 by the French Mathematician F. Marty [12] at the 8th Congress of Scandinavian Mathematicians where he defined the notion of hyperoperation on groups. In a classical algebraic structure, the binary operation of two elements of a set is again an element of the same set, while in an algebraic hyperstructure, the hyperoperation of two elements, is a subset of the same set. If this hyperoperation sends two elements to a singleton then the hyperoperation coincides with the classical binary operation.

Received: September 9, 2015

Published: February 2, 2016 (c) 2016 Academic Publications, Ltd. url: www.acadpubl.eu 
In literature, a number of different hyperstructure theories are widely studied since these represent a suitable and natural generalization of classical algebraic structures such as groups, rings and modules and for their applications to many areas of pure and applied mathematics and computer science.

In 2010, Davvaz and Mirvakili [8] defined a new class of $n$-ary multi-valued algebra called an $(m, n)$ - Krasner hyperring and defined a fundamental relation on the structure from which they obtained $(m, n)$-rings. Recently, Anvariyeh [2] carried out a research on $(m, n)$-ary hyper modules over $(m, n)$-hyperring and has been able to define a strongly compatible relation on the structure and determined a sufficient condition that the said relation is transitive.

In this paper, more characterization of prime and maximal hyper ideals not explored in [1] are presented. Moreover, Krasner ternary hyper field is defined and characterized in several ways.

\section{Preliminaries}

This section presents some definitions in hyperstructure theory and the preliminary concepts of this study.

Definition 2.1. [3] Let $H$ be a nonempty set and let $\wp^{*}(H)$ be the

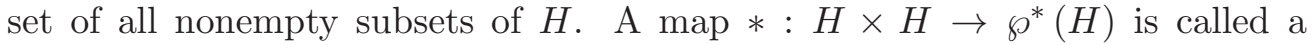
hyperoperation on the set $H$. The couple $(H, *)$ is called a hypergroupoid. If $A$ and $B$ are nonempty subsets of $H$, we define

i. $A * B=\bigcup_{a \in A, b \in B} a * b$

ii. $x * A=\bigcup_{a \in A} x * a$;

iii. $B * x=\bigcup_{b \in B} b * x$.

Example 2.2. Let $\mathbb{N}$ be the set of positive integers. Define a hyperoperation "*" on $\mathbb{N}$ by $x * y=\{x, y\}$ for all $x, y \in \mathbb{N}$. Then $(\mathbb{N}, *)$ is a hypergroupoid.

Definition 2.3. [3] A hypergroupoid $(H, *)$ is commutative if $a * b=b * a$ for all $a, b \in H$.

Definition 2.4. [3] A hypergroupoid $(H, *)$ is called a semihypergroup if for all $x, y, z \in H,(x * y) * z=x *(y * z)$. 
Definition 2.5. [3] A nonempty subset $B$ of a semihypergroup $H$ is called a subsemihypergroup of $H$ if $(B, *)$ is itself a semihypergroup.

Definition 2.6. [3] Let $(H, *)$ be a semihypergroup. Then $H$ is called a hypergroup if it satisfies the reproduction axiom, that is, $x * H=H * x=H$ for all $x \in H$.

Definition 2.7. [3] A nonempty set $H$ with a hyperoperation "+" is said to be a canonical hypergroup if the following are satisfied:

i. For every $x, y \in H, x+y=y+x$;

ii. for every $x, y, z \in H, x+(y+z)=(x+y)+z$;

iii. $\exists 0_{H} \in H$ (called the neutral element of $H$ ) such that $x+0_{H}=\{x\}$ and $0_{H}+x=\{x\}$ for all $x \in H$;

iv. for each $x \in H$ there exists a unique element denoted by $-x \in H$ such that $0_{H} \in[x+(-x)] \cap[(-x)+x]$;

v. for every $x, y, z \in H, z \in x+y$ implies $y \in-x+z$ and $x \in-y+z$.

The pair $(H,+)$ is called a canonical hypergroup.

Definition 2.8. [11] Let $R$ be a nonempty set. A ternary operation on $R$ is a map $f: R \times R \times R \rightarrow R$.

Example 2.9. Consider the set $\mathbb{Z}^{-}$of negative integers and "." is the usual multiplication on $\mathbb{Z}$. Then "." is a ternary operation in $\mathbb{Z}^{-}$which is not binary, since $\mathbb{Z}^{-}$is not closed under the binary product.

The remaining definitions and result in this section is taken from [5] unless otherwise specified.

Definition 2.10. A Krasner ternary hyperring is an algebraic structure $(R,+, \cdot)$ consisting of a nonempty set $R$, a hyperoperation "+" and a ternary multiplication "." satisfying the following:

i. $(R,+)$ is a canonical hypergroup;

ii. $(a \cdot b \cdot c) \cdot d \cdot e=a \cdot(b \cdot c \cdot d) \cdot e=a \cdot b \cdot(c \cdot d \cdot e)$;

iii. $(a+b) \cdot c \cdot d=a \cdot c \cdot d+b \cdot c \cdot d$;

iv. $a \cdot(b+c) \cdot d=a \cdot b \cdot d+a \cdot c \cdot d$;

v. $a \cdot b \cdot(c+d)=a \cdot b \cdot c+a \cdot b \cdot d$, 
vi. $a \cdot 0 \cdot 0=0 \cdot a \cdot 0=0 \cdot 0 \cdot a=0$.

for every $a, b, c, d, e \in R$. If without ambiguity, $a \cdot b \cdot c$ can be written as $a b c$ unless otherwise specified.

Example 2.11. Let $R=[0,1]$. We define a hyperaddition "+" on $R$ as follows:

$$
x+y= \begin{cases}{[0, x],} & \text { if } x=y \\ \{\max \{x, y\}\}, & \text { if } x \neq y\end{cases}
$$

and the ternary multiplication "." to be the ordinary multiplication. Then $(R,+, \cdot)$ is a Krasner ternary hyperring.

Definition 2.12. Let $(R,+, \cdot)$ be a Krasner ternary hyperring. A subset $S$ of $R$ is called a Krasner subternary hyperring if $(S,+, \cdot)$ is itself a Krasner ternary hyperring. A Krasner subternary hyperring $S$ of $R$ such that $S \neq R$ and $S \neq\{0\}$ is called proper subternary hyperring of $R$.

Definition 2.13. A subternary hyperring $I$ of a Krasner ternary hyperring $R$ is called a

i. right hyper ideal of $R$ if abi $\in I$ for all $a, b \in R$ and $i \in I$;

ii. left hyper ideal of $R$ if $i a b \in I$ for all $a, b \in R$ and $i \in I$;

iii. lateral hyper ideal of $R$ if $a i b \in I$ for all $a, b \in R$ and $i \in I$;

iv. two sided hyper ideal If $I$ is both a right and left hyper ideal;

v. hyper ideal if $I$ is left, right and lateral hyper ideal.

Theorem 2.14. (Hyper ideal Criterion) Let $R$ be a Krasner ternary hyperring. A nonempty subset $I$ of $R$ is a right [resp. left and lateral] hyper ideal of $R$ if and only if for all $i, j \in I$ and $a, b \in R$

i. $i+(-j) \subseteq I$,

ii. $a b i \in I$ [resp. $i a b \in R$ and $a i b \in R]$.

Definition 2.15. Let $R$ be a Krasner ternary hyperring. We say that $R$ admits an identity provided that there exists

$$
\left\{\left(e_{i}, f_{i}\right) \in R \times R \mid e_{i}, f_{i} \in R \text { and } n \in \mathbb{N}\right\}
$$


such that $\sum_{i=1}^{n} e_{i} f_{i} x=\sum_{i=1}^{n} e_{i} x f_{i}=\sum_{i=1}^{n} x e_{i} f_{i}=\{x\}$ for all $x \in R$. In this case, the Krasner ternary hyperring is said to be a Krasner ternary hyperring with identity. In particular, if there exists an element $1_{R} \in R$ such that $1_{R} 1_{R} x=$ $1_{R} x 1_{R}=x 1_{R} 1_{R}=x$ for all $x \in R$, then $1_{R}$ is called a unital element of $R$.

Theorem 2.16. Let $R$ be a Krasner ternary hyperring with unital element $1_{R}$ and $x \in R$. Then the principal

i. right hyper ideal generated by $x$ is given by

$$
\langle x\rangle_{r}=R R x=\bigcup\left\{\sum_{i=1}^{n} r_{i} s_{i} x \mid r_{i}, s_{i} \in R \text { and } n \in \mathbb{N}\right\},
$$

ii. left hyper ideal generated by $x$ is given by

$$
\langle x\rangle_{l}=x R R=\bigcup\left\{\sum_{i=1}^{n} x r_{i} s_{i} \mid r_{i}, s_{i} \in R \text { and } n \in \mathbb{N}\right\},
$$

iii. two-sided hyper ideal generated by $x$ is given by

$$
\langle x\rangle_{l}^{r}=R x R=\bigcup\left\{\sum_{i=1}^{n} r_{i} x s_{i} \mid r_{i}, s_{i} \in R \text { and } n \in \mathbb{N}\right\}
$$

iv. lateral hyper ideal generated by $x$ is given by

$$
\langle x\rangle_{c}=R x R+R R x R R=\bigcup\left\{\sum_{i=1}^{n} r_{i} x s_{i}+\sum_{j=1}^{m} p_{j} q_{j} x u_{j} v_{j}\right\}
$$

where $r_{i}, s_{i}, p_{j}, q_{j}, u_{j}, v_{j} \in R$ and $m, n \in \mathbb{N}$.

Lemma 2.17. Let $R$ be a Krasner ternary hyperring with unital element $1_{R}$ and $I$ a hyper ideal of $R$. If $1_{R} \notin I$, then $I \neq R$.

Remark 2.18. Let $R$ be a Krasner ternary hyperring, $I$ a hyper ideal of $R$, and $x, y \in R$. Then $x+I=y+I$ if and only if $x \in y+I$.

Theorem 2.19. Let $I$ and $J$ be right [ resp. left and lateral] hyper ideals of a Krasner ternary hyperring $R$ such that $I \subseteq J$. Then $J / I$ is a hyper ideal of $R / I$. 
Definition 2.20. Let $\left(R_{1},+, \cdot\right)$ and $\left(R_{2}, \oplus, *\right)$ be two ternary hyperrings. A mapping $\phi: R_{1} \rightarrow R_{2}$ is called a homomorphism if the following are satisfied:

i. $\phi(a+b)=\phi(a) \oplus \phi(b)$ for all $a, b \in H$;

ii. $\phi\left(a_{1} \cdot a_{2} \cdot a_{3}\right)=\phi\left(a_{1}\right) * \phi\left(a_{2}\right) * \phi\left(a_{3}\right)$ for all $a_{1}, a_{2}, a_{3} \in R_{1}$;

iii. $\phi\left(0_{R_{1}}\right)=0_{R_{2}}$.

Theorem 2.21. Let $f: R_{1} \rightarrow R_{2}$ be a homomorphism of Krasner ternary hyperrings. If $B$ is a hyper ideal of $R_{2}$, then

$$
f^{-1}(B)=\left\{a \in R_{1} \mid f(a) \in B\right\}
$$

is a hyper ideal of $R_{1}$ containing $\operatorname{Ker} f$.

Definition 2.22. Let $(R,+, \cdot)$ be a Krasner ternary hyperring. A nonempty subset $M$ of $R$ is said to be multiplicative in $R$ if $a b c \in M$ for all $a, b, c \in M$.

Theorem 2.23. [1] Let $S$ be a multiplicative subset of a Krasner ternary hyperring disjoint from a hyper ideal $I$. Then there exist a hyper ideal $P$ which is maximal in the set of all hyper ideals of $R$ disjoint from $S$ containing $I$. Furthermore, $P$ is prime.

\section{Main Results}

This section presents the results generated in this study.

Definition 3.1. A Krasner ternary hyperring $R$ is said to be commutative if for all $a, b, c \in R, a b c=a c b=b a c=b c a=c a b=c b a$.

Definition 3.2. Let $R$ be a Krasner ternary hyperring. A nonzero element $a$ of $R$ is said to be right invertible if there exist $b \in R$ such that $a t b=t$ for all $t \in R$, it is called left invertible if for all $t \in R$, there exists a nonzero $b \in R$ such that $b a t=t$ and lateral invertible if $a b t=t$. If $a$ is right, left and lateral invertible then $a$ is called invertible. The element $b$ is called the inverse of $a$.

Definition 3.3. A Krasner ternary hyperring $R$ is said to be zero divisor free if for all $a, b, c \in R$ with $a b c=0$ implies $a=0$ or $b=0$ or $c=0$.

Definition 3.4. Let $R$ be a Krasner ternary hyperring. A nonzero element $a$ of $R$ is called a unit in $R$ if $a$ is invertible. A Krasner ternary hyperring in which nonzero element is a unit is called a zero divisor free.

every 
Definition 3.5. A commutative zero divisor free Krasner ternary hyperring is called a Krasner ternary hyper field.

Definition 3.6. A hyper ideal $P$ of a Krasner ternary hyperring $R$ is called a prime hyper ideal of $R$, if for every hyper ideals $I, J$ and $K$ of $R$ satisfying $I J K \subseteq P$ implies either $I \subseteq P$ or $J \subseteq P$ or $K \subseteq P$.

Definition 3.7. A hyper ideal $M$ of a Krasner ternary hyperring $R$ said to be maximal if the following are satisfied:

i. $M \subset R$,

ii. if $I$ is a hyper ideal of $R$ such that $M \subset I$ then, $I=R$.

The next result guarantees the existence of a maximal hyper ideal in every Krasner ternary hyperring.

Theorem 3.8. Let $R$ be a non trivial Krasner ternary hyperring with unital element $1_{R}$. Then a maximal right [resp. left, lateral] hyper ideal always exists and every hyper ideal in $R$ is contained in a maximal right hyper ideal.

Proof. Let $I$ be a right hyper ideal in $R$ and

$$
\mathcal{S}=\left\{J_{i} \mid J_{i} \text { is a right hyper ideal of } R, I \subseteq J \text { and } J \neq R\right\}
$$

Then $\mathcal{S} \neq \varnothing$ since $I \in \mathcal{S}$. Partially order $\mathcal{S}$ by set inclusion, that is, $J_{1} \leq J_{2}$ if and only if $J_{1} \subset J_{2}$. Let $\mathcal{C}=\left\{C_{\omega} \mid \omega \in \Omega\right\}$ be a chain in $\mathcal{S}$ and $U=\bigcup_{\omega \in \Omega} C_{\omega}$. If $a, b \in U$, then there exists $C_{i}, C_{j} \in U$ such that $a \in C_{i}$ and $b \in C_{j}$. Since $\mathcal{C}$ is a chain, either $C_{i} \subset C_{j}$ or $C_{j} \subset C_{i}$. Without loss of generality, suppose that $C_{i} \subset C_{j}$. Then $a, b \in C_{j}$. Since $C_{j}$ is a right hyper ideal, by Theorem 2.14, $a+(-b) \subseteq C_{j}$ and $r s c \in C_{j}$ for all $r, s \in R$ and $c \in C_{j}$. Since $C_{j} \subseteq \bigcup_{\omega \in \Omega} C_{\omega}=U$, $a+(-b) \subseteq U$ and $r s c \in U$. Therefore, by Theorem 2.14, $U$ is a right hyper ideal of $R$. Since each $C_{\omega}$ is in $\mathcal{S}, C_{\omega} \neq R$ for all $\omega \in \Omega$. By Remark 2.17, $1_{R} \notin C_{\omega}$ for all $\omega \in \Omega$. Hence, $1_{R} \notin \bigcup_{\omega \in \Omega} C_{\omega}=U$. Hence, by Remark 2.17, $U \neq R$. Thus, $U \in \mathcal{S}$. Since $C_{\omega} \subseteq U$ for all $\omega \in \Omega, U$ is an upperbound of the chain $\mathcal{C}$. Thus, every chain in $\mathcal{S}$ has an upperbound in $\mathcal{S}$. So, by Zorn's Lemma, $\mathcal{S}$ has a maximal element. But a Maximal element of $\mathcal{S}$ is a maximal hyper ideal of $R$ that contains $I$.

Theorem 3.9. Let $M$ be a hyper ideal in a Krasner ternary hyperring $R$ with unital element $1_{R} \neq 0$. 
i. If $M$ is maximal and $R$ is commutative, then $R / M$ is a Krasner ternary hyper field.

ii. If $R / M$ is zero divisor free, then $M$ is maximal.

Proof.

i. Let $a+M \in R / M$ such that $a+M \neq M$. Then $M$ is properly contained in $\langle a\rangle_{l}+M$. By maximality of $M,\langle a\rangle_{l}+M=R$. Since $1_{R} \in R, 1_{R} \in\langle a\rangle+M$. Theorem 2.16, $1_{R} \in \sum_{i=1}^{n} a r_{i} s_{i}+m$ for some $m \in M, n \in \mathbb{N}$, and $r_{i}, s_{i} \in R$. Let $t+M \in R / M$. Then $t \in R$ and for some $m \in M$,

$$
\begin{aligned}
t & =1_{R} 1_{R} t \in\left[\left(\sum_{i=1}^{n} a r_{i} s_{i}\right)+m\right]\left[\left(\sum_{i=1}^{n} a r_{i} s_{i}\right)+m\right](t) \\
& =\left(a r_{1} s_{1}+\ldots+a r_{n} s_{n}+m\right)\left(a r_{1} s_{1}+\ldots+a r_{n} s_{n}+m\right)(t) \\
& =\left(a r_{1} s_{1} a r_{1} s_{1} t\right)+\ldots+\left(a r_{n} s_{n} a r_{1} s_{1} t\right)+\left(\operatorname{mar}_{1} s_{1} t\right) \\
& +\left(a r_{1} s_{1} a r_{2} s_{2} t\right)+\ldots+\left(a r_{n} s_{n} a r_{2} s_{2} t\right)+\left(\operatorname{mar}_{2} s_{2} t\right)+\ldots \\
& +\left(a r_{1} s_{1} a r_{n} s_{n} t\right)+\ldots+\left(a r_{n} s_{n} a r_{n} s_{n} t\right)+\left(\operatorname{mar}_{n} s_{n} t\right)+m m t
\end{aligned}
$$

$$
\begin{aligned}
& =\sum_{i, j} a\left(r_{i} s_{i} a r_{j} s_{j}\right) t+\sum_{i=1}^{n} \operatorname{mar}_{i} s_{i} t \\
& \subseteq a\left(\sum_{i, j}\left(r_{i} s_{i} a r_{j} s_{j}\right)\right)^{t+M+M .}
\end{aligned}
$$

Therefore, $t \in a b t+M$ for some $b \in \sum_{i, j} r_{i} s_{i} a r_{j} s_{j}$. By Remark 2.18, $a b t \in t+M$ for all $t+M \in R / M$. Hence,

$$
(a+M)(b+M)(t+M)=t+M \text {. }
$$

Therefore, $a+M$ is invertible. Since $R$ is commutative, it follows that $R / M$ is a Krasner ternary hyper field.

ii. Let $N$ be a hyper ideal of $R$ such that $M \subset N \subseteq R$. Then there exists an $n \in N \backslash M$. Since $R / M$ is zero divisor free, for all $M \neq a+M \in R / M$, there exists $b+M \in R / M$ such that $(a+M)(b+M)(t+M)=t+M$, 
for all $t+M \in R / M$. Thus, abt $+M=t+M$, for all $t \in R$. Hence, by Remark 2.18, abt $\in t+M$. In particular, since $1_{R} \in R$, there exists $b \in R$ such that $n b 1_{R} \in 1_{R}+M$. Hence, $n b 1_{R} \in 1_{R}+m$ for some $m \in N$. By Definition 2.7(v), $1_{R} \in n b 1_{R}+(-m)$. Since $n \in N, n b 1_{R} \in N$. Also, $m \in N$ since $M \subseteq N$. Consequently, $1_{R} \in n b 1_{R}+(-m) \subseteq N$. By Theorem 2.17, $N=R$. Therefore, $M$ is maximal.

Theorem 3.10. The following conditions in a commutative Krasner ternary hyperring $R$ with unital $1_{R} \neq 0$ are equivalent.

i. $R$ is a Krasner ternary hyper field,

ii. $R$ has no nontrivial hyper ideals,

iii. 0 is a maximal hyper ideal in $R$.

Proof. (i $\Rightarrow$ ii). Suppose to the contrary that $R$ has a nontrivial hyper ideal I. Then there exists $0 \neq t \in R \backslash I$. Let $i \in I$. Since $R$ is a Krasner ternary hyper field, there exists $b \in R$ such that $i b r=r$ for all $r \in R$. In particular, $i b t=t$. Since $I$ is a hyper ideal, $t=i b t \in I$, a contradiction. Therefore, $R$ has no proper hyper ideal.

(ii $\Rightarrow$ iii). Let $I$ be a hyper ideal of $R$ such that $0 \subset I \subseteq R$. Since $R$ has no proper hyper ideal, $0=I$ or $I=R$. Thus, $\{0\}$ is maximal.

(iii $\Rightarrow \mathrm{i}$ ). Suppose that $\{0\}$ is a maximal hyper ideal of $R$. Therefore, by Theorem 3.9(i), $R=R /\{0\}$ is a Krasner ternary hyper field.

Theorem 3.11. Let $R$ be a Krasner ternary hyperring with unital element $1_{R}, a, b, c$ are arbitrary elements of $R$ but fixed, and $P$ a hyper ideal of $R$. The following are equivalent:

i. $P$ is a prime hyper ideal of $R$,

ii. $\left\{a r_{i} b r_{j} c \mid r_{i}, r_{j} \in R\right\} \subseteq P$ if and only if $a \in P$ or $b \in P$ or $c \in P$,

iii. If $\langle a\rangle\langle b\rangle\langle c\rangle \subseteq P$, then either $a \in P$ or $b \in P$ or $c \in P$.

Proof. (i $\Rightarrow$ ii). Let $P$ be a prime hyper ideal of $R$. Suppose that either $a \in P$ or $b \in P$ or $c \in P$. Without loss of generality, suppose that $a \in P$. Since $P$ is a hyper ideal, $a r_{i} b r_{j} c \in P$ for all $r_{i}, r_{j} \in R$. So,

$$
\left\{a r_{i} b r_{j} c \mid r_{i}, r_{j} \in R\right\} \subseteq P \text {. }
$$


Conversely, assume that $\left\{a r_{i} b r_{j} c \mid r_{i}, r_{j} \in R\right\} \subseteq P$ and suppose that $a \notin P$ and $b \notin P$. Let $I=\langle a\rangle_{l}, J=\langle b\rangle_{l}^{r}$ and $K=\langle c\rangle_{r}$. Then $a \in I$ and $b \in J$. Hence, $I \nsubseteq P$ and $J \nsubseteq P$. Thus,

$$
\begin{aligned}
I J K & =(a R R)(R b R)(R R c) \\
& =a(R R R) b(R R R) c \\
& \subseteq a R b R c \\
& \subseteq P .
\end{aligned}
$$

Since $P$ is prime, then either $I=\langle a\rangle_{l} \subseteq P$ or $J=\langle b\rangle_{l}^{r} \subseteq P$ or $K=\langle c\rangle_{r} \subseteq P$. But $I \nsubseteq P$ and $J \nsubseteq P$. Therefore, $K \subseteq P$ and $c \in P$. (ii $\Rightarrow$ iii). Suppose that $\langle a\rangle\langle b\rangle\langle c\rangle \subseteq P$. Since $a \in\langle a\rangle, c \in\langle c\rangle$, and for all $r_{i}, r_{j} \in R, r_{i} b r_{j} \in\langle b\rangle$, it follows that $a\left(r_{i} b r_{j}\right) c \in\langle a\rangle\langle b\rangle\langle c\rangle \subseteq P$. Hence, $\left\{a r_{i} b r_{j} c \mid r_{i}, r_{j} \in R\right\} \subseteq P$. By (ii), either $a \in P$ or $b \in P$ or $c \in P$.

(iii $\Rightarrow \mathrm{i}$ ). Let $I, J$ and $K$ be hyper ideals of $R$ such that $I J K \subseteq P$. Suppose that $I \nsubseteq P$ and $J \nsubseteq P$. Then there exists $i \in I$ and $j \in J$ such that $i, j \notin P$. Since $\langle i\rangle \subseteq I$ and $\langle j\rangle \subseteq J$ and for all $k \in K,\langle k\rangle \subseteq K$. Thus, $\langle i\rangle\langle j\rangle\langle k\rangle \subseteq I J K \subseteq P$. By condition (iii), either $i \in I, j \in J$ or $k \in K$. Since $i \notin I$ and $j \notin J$, it follows that $k \in K$ and $K \subseteq P$. Consequently, $P$ is prime.

Theorem 3.12. A hyper ideal $P$ of a Krasner ternary hyperring $R$ is prime if and only if for every $a, b, c \in P^{c}$, there exist $r_{i}, r_{j} \in R$ such that $a r_{i} b r_{j} c \in P^{c}$.

Proof. Suppose that $P$ is a prime hyper ideal of $R$ and $a, b, c \in P^{c}$. Then $a, b, c \notin P$. By Theorem 3.11, $\left\{a r_{i} b r_{j} c \mid r_{i}, r_{j} \in R\right\} \nsubseteq P$. Thus, there exist $r_{i}, r_{j} \in R$ such that $a r_{i} b r_{j} c \notin P$. Therefore, $a r_{i} b r_{j} c \in P^{c}$. Conversely, let $a, b, c \notin P$. By assumption, there exist $r_{i}, r_{j} \in R$ such that $a r_{i} b r_{j} c \in P^{c}$, that is, $a r_{i} b r_{j} c \notin P$. Thus, $\left\{a r_{i} b r_{j} c \mid r_{i}, r_{j} \in R\right\} \nsubseteq P$. Therefore, by Theorem 3.11, $P$ is a prime hyper ideal.

Theorem 3.13. A maximal hyper ideal of a Krasner ternary hyperring $R$ with unital element $1_{R}$ is prime.

Proof. Let $R$ be a Krasner ternary hyperring, $M$ a maximal hyper ideal of $R$, and $a, b$ and $c$ are arbitrary elements of $R$ but fixed. Let $\left\{a r_{i} b s_{j} c \mid r_{i}, s_{i} \in R\right\} \subseteq$ $M$. Suppose that $a \notin M$ and $b \notin M$. Then $M \neq R$ and $M$ is properly contained in $\langle a\rangle_{l}+M$ and $\langle b\rangle_{l}^{r}+M$. Hence, $\langle a\rangle_{l}+M=R$ and $\langle b\rangle_{l}^{r}+M=R$, by maximality of $M$. By Theorem 2.16, $\langle a\rangle_{l}=i R R$ and $\langle b\rangle_{l}^{r}=R j R$. Thus, there exists $r_{i}, q_{i}, u_{j}, v_{j} \in R, n_{1}, n_{2} \in \mathbb{N}$ and $m_{1}, m_{2} \in M$ such that 


$$
\begin{aligned}
1_{R} \in\left(\sum_{i=1}^{n_{1}} a r_{i} q_{i}\right)+m_{1} \text { and } 1_{R} \in\left(\sum_{j=1}^{n_{2}} u_{j} b v_{j}\right)^{+}+m_{2} . \text { Hence, } \\
k=1_{R} 1_{R} k \in\left[\left(\sum_{i=1}^{n_{1}} a r_{i} q_{i}\right)+m_{1}\right]\left[\left(\sum_{j=1}^{n_{2}} u_{j} b v_{j}\right)^{+m_{2}}\right]^{(c)} \\
=\left(a r_{1} q_{1}+\ldots+a r_{n_{1}} q_{n_{1}}+m_{1}\right)\left(u_{1} b v_{1}+\ldots+u_{n_{2}} b v_{n_{2}}+m_{2}\right)(c) \\
=\sum_{i, j} a\left(r_{i} q_{i} u_{j}\right) b v_{j} c+\left(m_{1} m_{2} c\right) \\
\subseteq M .
\end{aligned}
$$

Therefore, by Theorem 3.11, $M$ is prime.

Lemma 3.14. Let $R$ be a Krasner ternary hyperring. If $P$ is a prime hyper ideal of $R$, then $P^{c}$ is a multiplicative subset of $R$.

Proof. Let $i, j, k \in P^{c}$. Then $i, j, k \notin P$. Since $P$ is prime, $i j k \notin P$. Thus, $i j k \in P^{c}$. Therefore, $P^{c}$ is multiplicative.

Proof. Let $I, J$ and $K$ be hyper ideals of $R$ such that $P \subset I, P \subset J$ and $P \subset K$. Since $P \cap M=\varnothing$ and $I \cap M \neq \varnothing$ implies $I \cap M \neq \varnothing, J \cap M \neq \varnothing$ and $K \cap M \neq \varnothing$. Thus, there exists $i \in I \cap M, j \in J \cap M$ and $k \in K \cap M$. Since $M$ is multiplicative, $i j k \in M$ therefore, $i j k \in I J K \cap M$. Since $M \cap P=\varnothing$, $i j k \notin P$ consequently, IJK $\nsubseteq P$. Therefore, $P$ is prime.

Theorem 3.15. Let $R$ be a Krasner ternary hyperring. Then the complement of every proper hyper ideal of $R$ contains a nonempty multiplicative subset of $R$ if and only if every proper hyper ideal of $R$ is properly contained in a prime hyper ideal.

Proof. Let $I$ be a proper hyper ideal in $R$ and $M$ a nonempty multiplicative subset of $R$ such that $M \subseteq I^{c}$. Then $I \cap M=\varnothing$. By Theorem 2.23, there exists a hyper ideal $P$ in $R$ such that $I \subset P, P \cap M=\varnothing$ and $J \cap M \neq \varnothing$ for every hyper ideal $J$ properly containing $P$. Therefore, by Theorem $2.23, P$ is prime. Conversely, suppose that $I$ is a proper hyper ideal of $R$ and $P$, a prime hyper ideal such that $I \subset P$. Thus, $P^{c} \subset I^{c}$. Since $P$ is prime, by Lemma 3.14, $P^{c}$ is multiplicative and $P^{c} \neq \varnothing$ since $P \neq R$. Therefore, $I^{c}$ contains a nonempty multiplicative set.

Theorem 3.16. Let $R$ and $S$ be Krasner ternary hyperrings, $Q$ a prime hyper ideal of $S$, and $f: R \rightarrow S$ be a homomorphism. Then $f^{-1}(Q)$ is a prime hyper ideal of $R$ which contains $\operatorname{Ker} \phi$. 
Proof. By Theorem 2.21, $f^{-1}(Q)$ is a hyper ideal of $R$ which contains Ker $\phi$. Suppose $x_{1}, x_{2}, x_{3} \in R$ such that $x_{1} x_{2} x_{3} \in f^{-1}(Q)$. Then $f\left(x_{1} x_{2} x_{3}\right) \in Q$. Since $f$ is a homomorphism and $Q$ is prime, either $f\left(x_{1}\right) \in Q, f\left(x_{2}\right) \in Q$, or $f\left(x_{3}\right) \in Q$. Hence, either $x_{1} \in f^{-1}(Q), x_{2} \in f^{-1}(Q)$, or $x_{3} \in f^{-1}(Q)$. Therefore, $f^{-1}(Q)$ is prime.

Theorem 3.17. Let $R$ be a Krasner ternary hyperring, $I$ a hyper ideal of $R$ and $P$ a prime hyper ideal of $R$ containing $I$. Then $P / I$ is a prime hyper ideal of $R / I$.

Proof. Since $P$ is prime, $P$ is properly contained in $R$. Thus, there exists $x \in R$ such that $x \notin P$. Since $I \subseteq P, x \notin I$. Thus $x+I \neq I$ and $x+I \notin P / I$. Hence $P / I \neq R / I$. On the other hand, by Theorem 2.19, $P / I$ is a hyper ideal of $R / I$. Let $x+I, y+I, z+I \in R / I$ such that $(x+I)(y+I)(z+I) \in P / I$. Then $x y z+I \in P / I$ and $x y z \in P$. Since $P$ is prime, either $x \in P, y \in P$, or $z \in P$. Thus, either $x+I \in P / I, y+I \in P / I$, or $z+I \in P / I$.

\section{References}

[1] Ameri R. and Norouzi A., Prime and Primary Hyper ideals in Krasner $(m, n)$-hyperrings, European Journal of Combinatorics, Vol. 34 (2013) pp 378-390.

[2] Anvariyeh, S.M., and Mirkavili, S., Canonical $(m, n)$-Hypermodules over Krasner $(m, n)$ ary Hyperrings, Iranian Journal of Mathematical Sciences and Informatics, Vol.7, No.2 (2012) pp 56-63.

[3] Asokkumar A. and Velrajan M., A Note on Isomorphism Theorems of Hyperrings, International Journal of Mathematics and Mathematical Sciences, Volume 2010, Hindawi Publishing House.

[4] Asokkumar A. and Velrajan M., Hyperring of Matrices Over a Regular Hyperring, Italian Journal of Pure and Applied Mathematics, No. 23, (2008).

[5] Castillo,J.R., and Vilela, J.P., Quotient and Homomorphism in Krasner Ternary Hyperrings, International Journal of Mathematical Analysis, Vol 8 No. 58,(2014), pp. 28452859.

[6] Davvaz B., Fuzzy hyperideals in Ternary Semihyperrings, Iranian Journal of Fuzzy Systems Vol. 6, No. 4, (2009).

[7] Davvaz B. and Leoreanu-Fotea V., Hyperring Theory and Applications, International Academic Press, Palm Harbor, Florida, USA, (2007).

[8] Davvaz B., and Mirvakili S., Relations on Krasner $(m, n)$-hyperrings, European Journal of Combinatorics, Vol. 31 (2010), pp 236-242.

[9] De Salvo M., Hyperrings and Hyperfields. Annales Scientifiques de Universite de Clermont-Ferrand II. No. 22, (1984).

[10] Lehmer D. H., A Ternary Analogue of Abelian Groups, American Journal of Mathematics, Vol. 54, No. 2., (1932). 
[11] Lister W. G., Ternary Rings, Transactions of the American Mathematical Society, Vol. 154, (1971).

[12] Marty F., Sur une Generalization de la Notion de Groupe, 8th Congres Math. Scandinaves, Stockholm. pp. 45-49, (1934).

[13] Rota R., Strongly Distributive Multiplicative Hyperrings, Journal of Geometry, Vol.39, No. 1, (1990). 
